\title{
Profiles of communication disorder in children with velocardiofacial syndrome: Comparison to children with Down syndrome
}

\author{
Nancy J. Scherer, $P h D^{1}$, Linda L. D'Antonio, $P h D^{2}$, and Jennifer R. Rodgers, $M S^{1}$
}

\begin{abstract}
Purpose: To describe communication profiles in children with velocardiofacial syndrome (VCFS) compared with children with Down syndrome. Methods: Four children with VCFS and four children with Down syndrome underwent cognitive and speech and language assessment. Results: Communication profiles of children with Down syndrome showed a flat profile, indicating all measures were similar and delayed relative to chronological age. Children with VCFS showed vocabulary, pattern of sound types, and Mean Babbling Length below cognitive and other language ages. Conclusion: Communication profiles of children with VCFS differed qualitatively and quantitatively from children with Down syndrome and support the hypothesis that some children with VCFS present with a profile of communication impairment that may be distinctive to the syndrome. Genetics in Medicine, 2001:3(1):72-78.
\end{abstract}

Key Words: velocardiofacial syndrome, Down syndrome

Communication impairment is one of the prominent features of velocardiofacial syndrome (VCFS). ${ }^{1-3}$ However, the literature concerning speech and language characteristics in VCFS has been composed primarily of descriptive, retrospective reports, clinical audits, case studies, and short summary statements. Only one longitudinal study has been reported, ${ }^{4}$ and there has been little information comparing the impairments observed in children with VCFS with other clinical populations. In a longitudinal study by Scherer et al., ${ }^{4}$ a group of children with VCFS was followed from 6 to 30 months of age and compared with comparison groups of children with cleft lip and palate, isolated cleft palate, and typically developing children. The children with VCFS showed significant differences in receptive language, expressive language and speech sound acquisition compared with the other three comparison groups. This study suggested that the patterns of language and speech deficits in children with VCFS were distinctive from the development observed in the comparison groups and that differences observed in young children with VCFS were not due solely to the affects of cleft palate or middle ear pathology associated with clefting.

A second study by D'Antonio et al. ${ }^{5}$ compared the speech patterns of a group of children with VCFS to a group of children with speech impairment and some phenotypic character-

\footnotetext{
From the ${ }^{1}$ Department of Communicative Disorders, East Tennessee State University, Johnson City, Tennessee; ${ }^{2}$ Department of Surgery, Loma Linda University School of Medicine, Loma Linda, California.

Nancy J. Scherer, PhD, Box 70634, Dept. Of Communicative Disorders, East Tennessee, State University, Johnson City, TN 37614.

Received: August 14, 2000.

Accepted: October 18, 2000.
}

istics of VCFS without a deletion at 22q11.2. The findings of this study showed that young children with VCFS had significant deficits in speech performance beyond that of the comparison group. Scherer et al. ${ }^{4}$ and D'Antonio et al. ${ }^{5}$ interpreted the evidence from these two studies to suggest that some children with VCFS demonstrate a communication profile that may be distinctive to this syndrome. However, further studies are needed to compare the profiles of speech and language impairment in children with VCFS to other groups with known profiles of speech and language impairment.

Children with Down syndrome demonstrate some similarities to children with VCFS, making them an important comparison group. These children have similar early developmental histories such as hypotonia, feeding difficulties, middle ear pathology, and developmental delays. Further, children with Down syndrome provide an excellent comparison group because there are considerable research data available on the communication profiles of these children. Miller ${ }^{6}$ describes three distinct profiles of impairment, that take into account mental age, language comprehension, and language production from 20 years of research on children with Down syndrome. These profiles are based on a large, longitudinal database of communicative development of children with Down syndrome. Miller's work has become the standard to which profiles of other children with syndromes are compared (e.g., Williams, fragile X).

The purpose of the present study is to describe the communication profiles of children with VCFS and to compare these profiles with those reported for children with Down syndrome. Such comparison provides further data regarding the hypothesis that the profile of communication impairment in children with VCFS may be distinctive to this syndrome. 
Table 1

Age, cleft type, gender, receptive age scores from the Sequenced Inventory of Communicative Development-R, mental scale score, and mental age score from the Bayley Scales of Infant Development-2 and hearing status for the children with velocardiofacial syndrome (VCFS) and children with Down syndrome

\begin{tabular}{|c|c|c|c|c|c|c|c|}
\hline Subjects & $\begin{array}{c}\text { Age } \\
\text { (months) }\end{array}$ & Cleft type & Gender & $\begin{array}{c}\text { Receptive } \\
\text { language age }\end{array}$ & $\begin{array}{c}\text { Bayley } \\
\text { Mental Scale } \\
\text { score }\end{array}$ & $\begin{array}{l}\text { Mental age } \\
\text { (months) }\end{array}$ & Hearing \\
\hline \multicolumn{8}{|l|}{ VCFS } \\
\hline 1 & 30 & SMCP & M & 20 & 63 & 19 & Pass $^{\star}$ \\
\hline 2 & 30 & ICP & M & 20 & 50 & 15 & Pass $^{\star}$ \\
\hline 3 & 30 & $\mathrm{NC}$ & $\mathrm{F}$ & 16 & 81 & 24 & Pass \\
\hline \multicolumn{8}{|c|}{ Down syndrome } \\
\hline 1 & 24 & $\mathrm{NC}$ & M & 20 & 50 & 12 & Pass $^{\star}$ \\
\hline 2 & 25 & $\mathrm{NC}$ & $\mathrm{M}$ & 20 & 62 & 16 & Pass \\
\hline
\end{tabular}

Asterisks indicate PE tubes. SMCP, submucous cleft palate; ICP, isolated cleft palate; NC, no identified cleft. Pass indicates hearing sensitivity at $20 \mathrm{~dB}$ or better bilaterally at $500 \mathrm{~Hz}, 1,2$, and $4 \mathrm{~K}$, and normal middle ear function.

\section{METHODS}

\section{Subjects}

Following institutional review board approval and obtaining informed consent, four children with VCFS and four children with Down syndrome were studied. The children were individually matched for language comprehension level (within 4 months) based on the receptive language age scores of the Sequenced Inventory of Communicative Development-R (SICD-R). ${ }^{7}$ All children were recruited from upper east Tennessee. Exclusionary criteria for the study included children with (1) severe motor impairment, (2) sensorineural hearing loss or uncorrected visual impairment, or (3) chronic medical conditions that required frequent hospitalizations (e.g., gastrostomy, tracheostomy). All children were evaluated by a geneticist. The diagnosis of VCFS was confirmed through fluorescence in situ hybridization (FISH). ${ }^{8}$ Trisomy 21 was confirmed though chromosomal studies.

Table 1 presents the age, gender, receptive language age scores, developmental scores, cleft type (if any), and hearing status for all children. The children with VCFS were referred for this study by Tennessee Early Intervention and the Regional Cleft Palate Team, Johnson City, TN. The children with Down syndrome were recruited from Tennessee Early Intervention and matched to the children with VCFS based on the their receptive language age, which was approximately 16 to 20 months, and socioeconomic status. ${ }^{9,10}$ All of the children with VCFS had a chronologic age of 30 months while two of the children with Down syndrome were 24 and 25 months and two were 54 and 51 months of age.

\section{Procedures}

\section{Cognition}

The Bayley Scales of Infant Development-211 (BSID-2) were administered to each child. Standard scores and age scores were computed from the mental scale.

\section{Standardized language test}

Each child was given the Sequenced Inventory of Communicative Development-Revised. ${ }^{7}$ Receptive and expressive language ages scores were derived from the results.

\section{Language sample}

A 30-minute language sample was collected to assess expressive language use in a naturalistic home setting. This measure provides an assessment of language skills not available in standardized tests. The language samples were analyzed using the Systematic Analysis of Language Transcripts (SALT). ${ }^{12}$ The measures taken from the SALT were (1) the number of different words used in the conversation, (2) the total number of words used, and (3) Mean Length of Utterance (MLU).

\section{Parent questionnaire}

The MacArthur Communicative Development Inventory (CDI) was completed by the parents. ${ }^{13}$ In addition to word combinations and word endings, this measure assesses the children's vocabulary size based on their language use at home. The number of different words reported by the parent was taken from this measure.

\section{Speech sound production}

An analysis of the children's sound system was completed. The analysis examined all sounds produced by the children 
without reference to the adult model. An inventory of consonant sounds was taken from phonetic transcription of the sounds and words used in the language sample. Speech sounds were considered present in the children's consonant inventory if they appeared at least twice in the language sample, regardless of whether it was used correctly in standard adult form. ${ }^{14}$

Many of the children in this study used few true words; therefore, Mean Babbling Level (MBL) was calculated to provide a description of the phonetic complexity of their vocalizations. The MBL was calculated by classifying the children's vocalizations into one of three categories..$^{15}$ Level 1 consisted of vocalizations that were vowels only $(\mathrm{V})$, consonant-vowel (CV) or VC combinations, in which the consonants used were glottal (h or glottal stop) or glides (w, y). Level 2 consisted of use of all other consonants in $\mathrm{CV}, \mathrm{VC}$, or CVCV with the same consonant (e.g., mama). Level 3 consisted of vocalizations that contained at least two different consonants (e.g., bada). MBL was calculated by weighting the vocalizations by level (e.g., 1, 2, or 3 points) and dividing by the total number of vocalizations according to the method described by Stoel-Gammon. ${ }^{15}$

\section{Reliability}

Inter- and intraobserver reliability was completed on $20 \%$ of the language sample and phonetic transcriptions for each child. Point-by-point inter- and intraobserver agreement was 94 and $95 \%$, respectively.

\section{RESULTS}

\section{Cognition}

Table 2 shows the mental scale score (mean of 100 and standard deviation of 15), derived from the BSID-2, and mental age score. The children in the VCFS group showed scale scores that ranged from 50 to 81 . The children with Down syndrome had

Table 2

Developmental ages for the cognitive (nonlanguage) and language items of the Bayley Scales of Infant Development-2 for the children with velocardiofacial syndrome (VCFS) and children with Down syndrome

\begin{tabular}{lccc}
\hline & $\begin{array}{c}\text { Mental } \\
\text { age } \\
\text { Subjects }\end{array}$ & $\begin{array}{c}\text { Cognitive } \\
\text { age } \\
\text { standard scale }\end{array}$ & $\begin{array}{c}\text { Language } \\
\text { age } \\
\text { (months) }\end{array}$ \\
\hline
\end{tabular}

\begin{tabular}{lllll}
\hline VCFS & & & & \\
1 & 63 & 19 & 26 & 14 \\
2 & 50 & 15 & 19 & 14 \\
3 & 81 & 24 & 26 & 17 \\
4 & 75 & 23 & 26 & 14 \\
& & & & \\
Down syndrome & & & & \\
1 & 50 & 15 & 14 & 16 \\
2 & 62 & 16 & 13 & 18 \\
3 & 50 & 29 & 26 & 30 \\
4 & 45 & 23 & 22 & 25 \\
\hline
\end{tabular}

scale scores that ranged from 45 to 62 . Mental ages for both groups were similar, ranging from 15 to 24 months for the children with VCFS and 12-27 months for the children with Down syndrome. However, the mental scale of the BSID-2 includes many speech and language items. Therefore, the cognitive and language items of the BSID-2 were examined separately using the item analysis provided in the BSID-2 administration manual. The children with Down syndrome showed similar performance across cognitive and language domains. In contrast, the children with VCFS showed differences between cognitive and language performance, with language ages 5 to 12 months below performance in the nonlanguage cognitive domain. The mean chronological age of the children with VCFS was 30 months while the mean age of the children with Down syndrome was 38.5 months.

\section{Language}

Table 3 shows the data collected in the language sample and parent questionnaire. The children with VCFS used fewer different words in the language sample than the children with Down syndrome. Additionally, the total number of words used during conversation, an indication of general talkativeness, showed fewer words used during interaction for the children with VCFS than the children with Down syndrome. Further, the size of the children's vocabulary, as indicated by the parents, showed differences in overall vocabulary used in the home with the children with VCFS lagging behind the children with Down syndrome.

\section{Speech production}

Speech sound production was examined from the words and vocalizations used in the language sample. Table 4 displays the sound inventory for each child. The sounds are organized

\section{Table 3}

Language data from the language sample (Systematic Analysis of Language Transcripts, SALT) analysis and parent questionnaire

\begin{tabular}{lcccc}
\hline & \multicolumn{3}{c}{ SALT } & \\
Subjects & $\begin{array}{c}\text { Different } \\
\text { words }\end{array}$ & $\begin{array}{c}\text { CDI } \\
\text { frequency }\end{array}$ & MLU & $\begin{array}{c}\text { CDI } \\
\text { vocabulary size }\end{array}$ \\
\hline
\end{tabular}

\begin{tabular}{crrrr}
\hline VCFS & & & & \\
1 & 7 & 15 & 1.0 & 105 \\
2 & 2 & 3 & $*$ & 5 \\
3 & 2 & 5 & $*$ & 5 \\
4 & 4 & 5 & $*$ & 15
\end{tabular}

Down syndrome

\begin{tabular}{lrrrc}
1 & 22 & 52 & 1.0 & 56 \\
2 & 1 & 1 & $*$ & 40 \\
3 & 29 & 101 & 1.5 & 611 \\
4 & 43 & 95 & 1.6 & 180 \\
\hline \\
Asterisks indicate insufficient words to calculate MLU. MLU, Mean Length of \\
Utterance; CDI, MacArthur Communicative Development Inventory.
\end{tabular}


Table 4

Consonant inventory for the children with velocardiofacial syndrome (VCFS) and children with Down syndrome

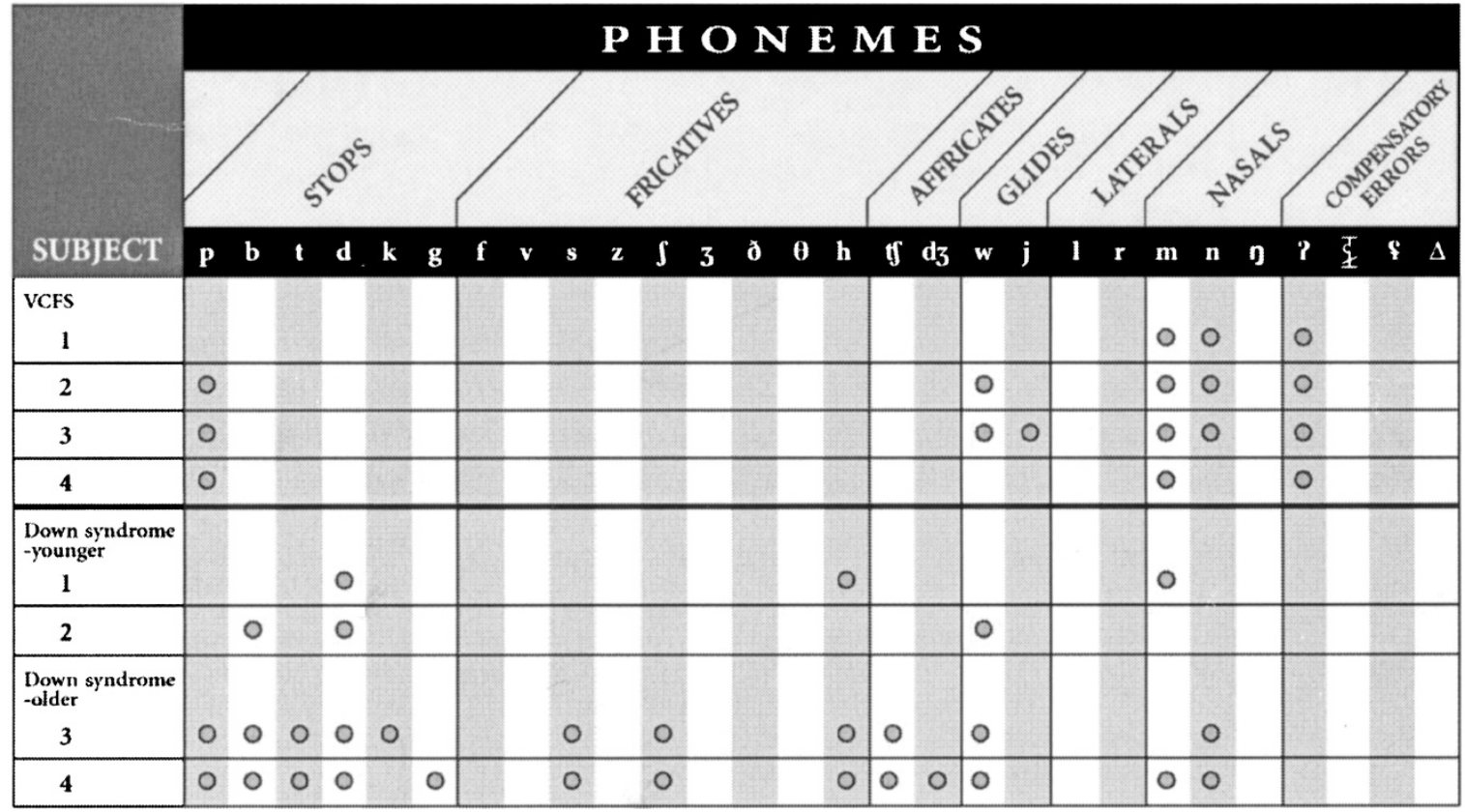

Stops $=$ oral sounds made by closing the vocal tract to block the air stream; fricatives $=$ oral sounds made with a continuous air stream; affricates $=$ oral sounds made with both stop and fricative; glides = oral consonant sounds that are made by moving from a partly constricted vocal tract to an open vocal tract; laterals = oral sounds made by letting air escape around the sides of the tongue; nasals = sounds made with an open velopharyngeal port; and compensatory errors = sounds made in the nasal cavity, pharynx, or larynx that are substituted for oral sounds.

from sounds made in the front of the mouth to sounds produced in the back of the vocal tract. The children with VCFS used fewer sounds than the children with Down syndrome. However, the inventories of the children with VCFS appeared most similar to the inventories of the younger children with Down syndrome. However, there were several differences in the sounds present in the inventories. The children with Down syndrome used a number of different sound classes, while the children with VCFS were limited in the sound categories and used sounds in the front and back of the vocal tract. Also, the children with VCFS used glottal stops while this sound was not used by children with Down syndrome.

Table 5 shows the children's speech sound production when compared to the adult model. The number of consonants used by the children with VCFS ranged from 3 to 5 consonants, while the younger children with Down syndrome used 3-4 consonants, and the older children with Down syndrome used 11 consonants. Since many of the children in this study did not use many words, their vocalizations were analyzed for complexity using Mean Babbling Level (MBL). ${ }^{15}$ All of the children with VCFS had MBLs of 1, indicating the least complex level of development, characterized by glottal and glide consonants used in consonant-vowel (CV) or VC combinations. The children with Down syndrome had MBLs of 1.5 to 2.4, indicating use of true consonants in $\mathrm{CV}, \mathrm{VC}$, and $\mathrm{CVCV}$ sequences with the same consonants (e.g., mama) and different consonants (e.g., maba).
Table 5

Number of consonants and Mean Babbling Level (MBL) for the children with velocardiofacial syndrome (VCFS) and Down syndrome

\begin{tabular}{ccc}
\hline Subjects & $\begin{array}{c}\text { No. of } \\
\text { consonants }\end{array}$ & MBL \\
\hline VCFS & 3 & 1.0 \\
1 & 4 & 1.0 \\
2 & 5 & 1.0 \\
3 & 4 & 1.0
\end{tabular}

\begin{tabular}{crl} 
Down syndrome & & \\
1 & 4 & 1.5 \\
2 & 3 & 1.5 \\
3 & 11 & 2.2 \\
4 & 11 & 2.4 \\
\hline
\end{tabular}

\section{Profiles}

To summarize the preceding results, communication profiles were developed from the data. In order to place the speech and language measures on a single scale, each score was converted to an age score based on test norms and/or developmental norms. Figure 1 shows the communicative profiles devel- 


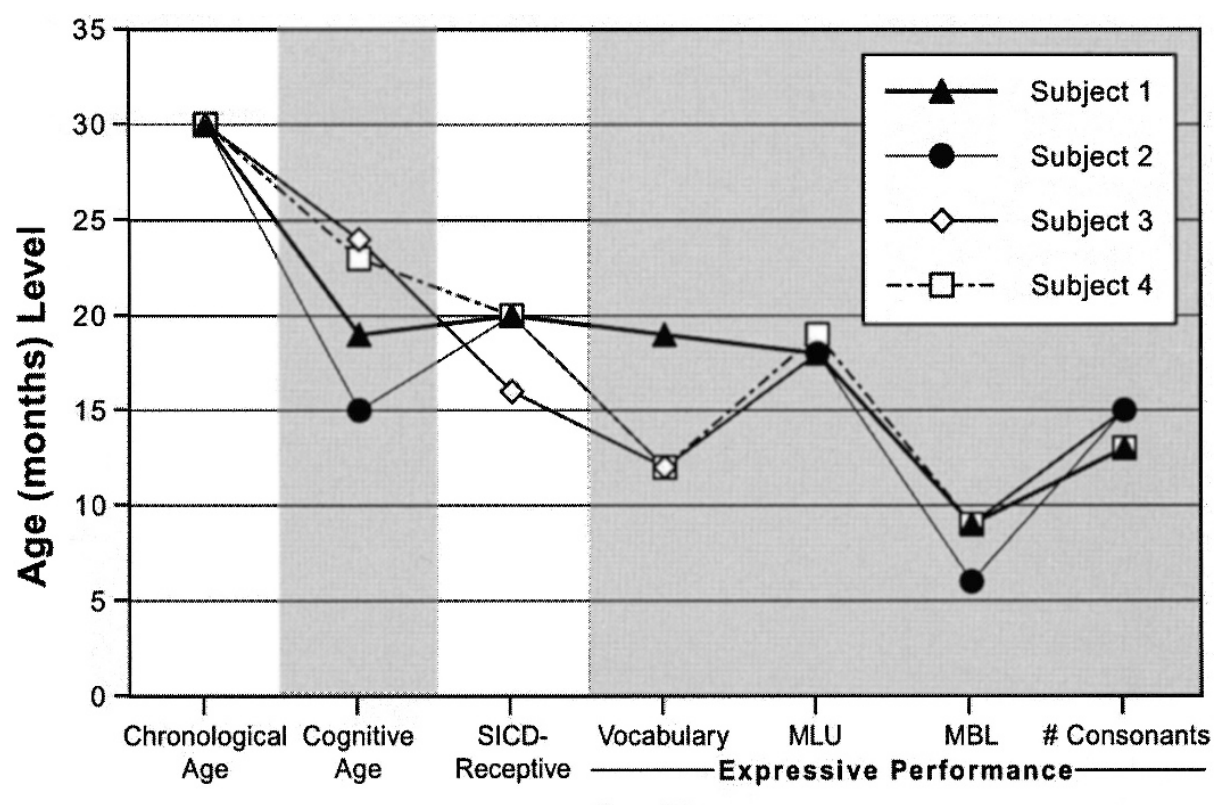

\section{Profiles}

Fig. 1 The communicative profiles for the children with velocardiofacial syndrome.

oped for the children with VCFS. Chronological, mental, and cognitive (nonlanguage) ages are provided as a comparison with language and speech domains. Receptive language was matched at 16-20 months of age. Expressive language parameters of vocabulary (CDI norms), MLU, ${ }^{16}$ and speech measures, including the number of consonants, ${ }^{15,17}$ and MBL ${ }^{14,15}$ are provided for each child. The children with VCFS showed similar profiles with weaknesses in vocabulary, the pattern, and distribution of sound types, and MBL.

Figure 2 shows the communication profiles of the children with Down syndrome. While all the children in this study were matched for language comprehension level, the children with

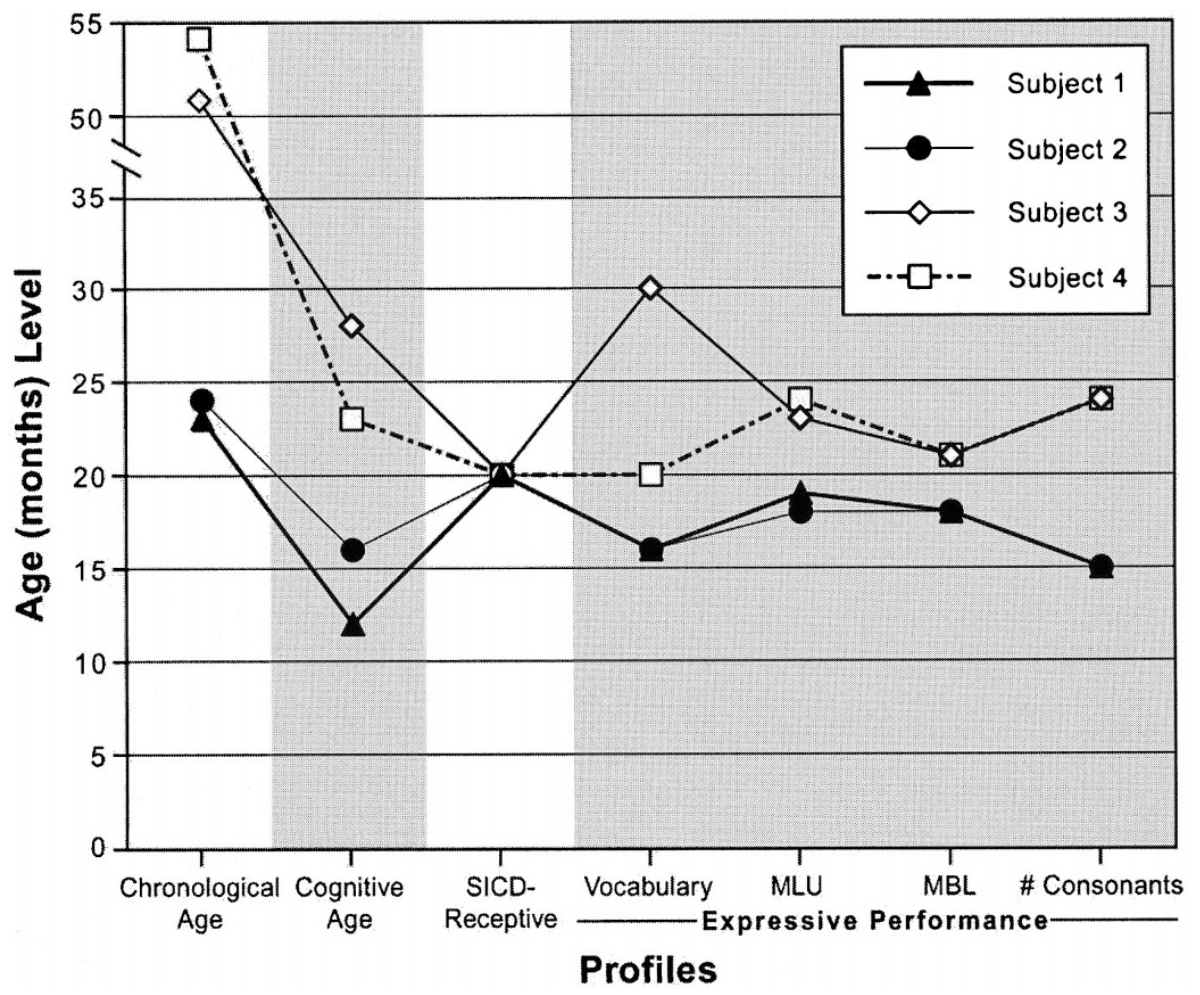

Fig. 2 Communicative profiles for the children with Down syndrome. 
Down syndrome who were older chronologically showed relative strengths in vocabulary and speech sound measures when compared to the younger children with Down syndrome and the children with VCFS.

Comparison of the communication profiles indicates that children with Down syndrome showed a flat profile, indicating receptive language, expressive language, speech and cognitive ages were similar and delayed relative to chronological age. In contrast, the children with VCFS showed vocabulary and MBL performance below that of other language and cognitive ages.

\section{DISCUSSION}

Previous studies have found differences between the communication profiles of children with VCFS and children with palatal clefting and children with similar phenotypic characteristics without VCFS. ${ }^{4,5}$ The purpose of the present study was to provide further data regarding the nature of the profile of communication impairment. The present results indicate that the communicative profiles of the children with VCFS differed from children with Down syndrome, when groups were matched for language comprehension.

The speech and language characteristics of children with Down syndrome have been well documented. Miller ${ }^{6}$ proposed three profiles of communication development specific to these children. Profile 1, representing 34\% of the children with Down syndrome, showed a pattern of language comprehension and production that was commensurate with mental age. Profile 2 , containing $64 \%$ of the children, showed similar mental age and language comprehension but reduced language production performance. Profile 3, representing $2 \%$ of the children, showed a significant delay in both language comprehension and production far below their mental age performance. The children with Down syndrome in this study showed performance similar to Miller's profile 1, indicating receptive language, expressive language, and speech performance was commensurate with mental age. The communicative performance of the children with VCFS showed a different pattern. The children with VCFS showed receptive language equal to or below mental age and reduced expressive language and speech production performance. These findings are consistent with the findings of Scherer et al. ${ }^{4}$ showing a difference in the profiles of speech and language impairment in children with VCFS compared with children with cleft palate. In addition to the children with VCFS showing a different profile from the children with Down syndrome, they showed a greater severity of impairment in vocabulary use and MBL from the children with Down syndrome. Inspection of the phonetic repertoire for the two groups shows a pattern of restricted place and manner of articulation for children with VCFS that relies on sounds made in the front and back of the vocal tract with no mid-vocal tract productions. This pattern of restricted place of articulation was observed previously when comparing the phonetic repertoires of children with VCFS compared with children having speech impairment and some phenotypic overlap with VCFS. ${ }^{5}$ The communicative profiles of the chil- dren with VCFS was differentiated from the children with Down syndrome by vocabulary, place of articulation, and MBL.

The data from this study interpreted collectively with previous reports ${ }^{4,5}$ support the hypothesis that at least some children with VCFS present with a communication profile which is distinctive to the syndrome with communication profiles that are characterized by severe deficits in early vocabulary acquisition and speech sound production. The data from the studies of comparison populations are compelling because they represent comparisons with children who demonstrate a number of the conditions associated with VCFS and yet their communication profiles differ. For example, the children with cleft lip and palate share the impairment in palate structure and middle ear pathology that could contribute to early speech and language deficits in children with VCFS. Similarly, the children with isolated clefts have the palatal impairments and pervasive receptive/expressive language deficits as well as middle ear issues. The children with phenotypic characteristics similar to VCFS but without the deletion provide a comparison to children with severe speech and resonance deficits. And the children with Down syndrome demonstrate early feeding difficulties, hypotonia, and developmental delays that could also contribute to early communication impairments. Despite the similarities in predisposing conditions and underlying comorbidity, the children with VCFS consistently differ from children in the comparison groups in the profile of communication impairment. While the numbers of subjects studied in each of these investigations have been small, the consistent, repeated pattern of a different profile of communication impairment for children with VCFS is robust.

\section{Clinical implications}

As with the previous studies that documented differences between the profiles of children with VCFS compared with children with cleft palate or phenotypic overlap, the present study reports findings which are relevant to clinical practice. First, the performance on the nonlanguage and language items of the cognitive assessment point to the importance of separating nonlanguage, cognitive performance from language performance when deriving estimates of cognitive function. For young children, this can be accomplished using the BSID-2, which has an item analysis that separates these functions and yields age scores

Secondly, the data from this study demonstrate the value of communication profiling to assist in characterizing the pattern of speech and language impairment in a given child and for developing appropriate treatment plans. For example, the children with VCFS in this study showed deficits in vocabulary acquisition and speech sound production. Methods for treating these primary deficits may then be developed to optimize progress. While there are few data available on early speech and language treatment methods in children with VCFS, a study by Scherer ${ }^{18}$ found that speech sound production in children with cleft palate could be enhanced by a treatment method that focused on vocabulary intervention. As vocabulary improved, speech sound inventories 
increased. Studies of other populations of young children with speech and language impairments has shown similar benefits of early vocabulary intervention. ${ }^{19,20}$ Additionally, communication profiling could assist in determining the impact of different treatments, particularly in the current absence of treatment data for young children with VCFS.

A third clinical implication of the present data set involves MBL. The data indicate that MBL appeared to be a useful tool in discriminating between the children with VCFS and children with Down syndrome. This measure has been used with other children with speech and language impairments. For example, a study by Scherer et al. ${ }^{21}$ which assessed the predictive value of vocalizations in children with cleft palate and showed that MBL, taken at 6 and 12 months of age, was a predictor of speech production at 30 months of age. The present data regarding the severe restriction in MBL for children with VCFS suggest that MBL may be a powerful predictive tool which should be considered as part of a routine clinical battery for young children with VCFS.

\section{Limitations of the study}

This study was a preliminary investigation of the communication profiles of children with VCFS compared to children with Down syndrome. While the study presents data to support further research using a comparison group of children with Down syndrome, several limitations were apparent. First, the small numbers of subjects in both groups preclude generalization to the population as a whole. Specifically, variation in chronological age and lack of a complete gender match in the Down groups is problematic. However, the results of the study indicate the potential benefits of a study with larger numbers of children with Down syndrome to investigate whether young children with Down syndrome show the profile exhibited by the children in this study. Since the children demonstrated only one of the three possible profiles, generalization to a larger population is uncertain until a larger study is conducted.

\section{Acknowledgments}

Portions of this research were supported by National Institute on Deafness and Other Communicative Disorders grant DC02301-01A1.

\section{References}

1. Shprintzen R, Goldberg RB, Lewin ML, Sidoti EJ, Berkman MD, Argamaso RV, Young D. A new syndrome involving cleft palate, cardiac anomalies, typical facies and learning disabilities: velo-cardio-facial syndrome. Cleft Palate J 1978;15:56-62.

2. Golding-Kushner K, Weller, Shprintzen R. Velo-cardio-facial syndrome: language and psychological profiles. J Craniofac Genet Dev Biol 1985;5:259-266.

3. Gerdes M, Solot C, Wang P, Moss E, LaRossa D, Randall P, Goldmuntz E, Clark BJ, Driscoll D, Jawad A, Emanuel B, McDonald-McGinn, Batshaw M, Zackai E. Cognitive and behavior profile of preschool children with chromosome 22q11.2 deletion. Am J Med Genet 1999;85:127-133.

4. Scherer NJ, D'Antonio L, Kalbfleisch J. Early speech and language development in children with velocardiofacial syndrome. Am J Med Genet 1999;88:714-723.

5. D'Antonio L, Scherer NJ, Miller L, Kalbfleisch J, Bartley J. Analysis of speech characteristics in children with velocardiofacial syndrome (VCFS) and children with phenotypic overlap without VCFS. Cleft Palate Craniofac J In press.

6. Miller J. Profiles of language development in children with Down syndrome. In: J. Miller, Leddy M, Leavitt L, editors. Improving the communication of people with Down syndrome. Baltimore: Brookes Publishing, 1999

7. Hedrick DL, Prather EM, Tobin AR. Sequenced inventory of communicative development-revised. Seattle: University of Washington Press, 1976.

8. Scambler PJ, Kelly D, Lindsay E, Williamson R, Goldberg R, Shprintzen R, Wilson DI, Goodship J, Cross IE, Brun J. Velo-cardio-facial syndrome associated with chromosome 22 deletions encompassing the DiGeorge locus. Lancet 1992;339:11381139

9. Caselli M, Vicari S, Longobardi E, Lame, Pizzoli C, Stella G. Gestures and words in early development of children with Down syndrome. J Speech Lang Hear Res 1998; 41:1125-1135

10. Facon B, Gruber J, Gardez C. Chronological age and receptive vocabulary of persons with Down syndrome. Psychol Rep 1998;82:723-726.

11. Bayley N. Bayley scales of infant mental development-revised. New York: Psychological Corporation, 1993.

12. Miller J, Chapman R. Systematic analysis of language transcripts. Madison, WI: Language Analysis Laboratory, University of Wisconsin, 1999.

13. Fenson L, Dale P, Reznick S, Thal D, Bates E, Hartung J, Pethick S, Reilly J. The MacArthur communicative development inventories. San Deigo, CA: Singular Publishing, 1993.

14. Stoel-Gammon C. Phonetic inventories, 15-24 months: a longitudinal study. $J$ Speech Hear Res 1985;28:505-512.

15. Stoel-Gammon C. Prespeech and early speech development of two late talkers. First Lang 1989;9:207-224.

16. Miller J, Chapman R. The relation between age and mean length of utterance in morphemes. J Speech Hear Res 1981;24:151-161.

17. Smith B, Stoel-Gammon C. A longitudinal study of the development of stop consonant production in normal and Down syndrome children. J Speech Hear Disord 1983;48:114-118.

18. Scherer NJ. The speech and language status of toddlers with cleft lip and/or palate following early vocabulary intervention. Am J Speech Lang Pathol 1999;8:31-40.

19. Paul R, Jennings P. Phonological behavior in toddlers with slow expressive language development. J Speech Hear Res 1992;35:99-107.

20. Rescorla L, Ratner M. Phonetic profiles of toddlers with specific expressive language impairment. J Speech Hear Res 1996;39:153-165.

21. Scherer NJ, Williams AL, Kalbfleisch J. Profiles of early and later vocalizations in children with cleft lip and/or palate and children without clefts. Paper presented at the Symposium on Research in Child Language Disorders, University of WisconsinMadison, June 5-7, 1999. 